\title{
The respiratory ciliary motion produced by dynein activity alone: A computational model of ciliary ultrastructure
}

\author{
Duanduan Chen*, Jun Ren, Yuqian Mei and Yuanqing Xu \\ School of Life Science, Beijing Institute of Technology, Beijing, China
}

\begin{abstract}
.
BACKGROUND: Respiratory ciliary motion is enabled by dynein/microtubule activity. Current observation techniques can hardly capture the dynein activation pattern in moving cilia. Here we introduce a computational model to mimic the ciliary ultrastructure and simulate the dynein-driven ciliary motion.

METHODS: A three-dimensional model is established to mimic the " $9+2$ " ciliary ultrastructure. The dynein force is simulated as point loads embedded along the microtubules. The dynein-triggered ciliary motion is solved by using the Finite Element Method along with grid deformation techniques.

RESULTS: By comparing the simulated ciliary movement to the observation results, the rationality of different dynein activity hypotheses are evaluated and the dynein activation pattern that can produce the planar beating of lung cilia is proposed. The results also reveal that the dynein force alone can only generate longitudinal microtubule sliding and ciliary bending; to produce the ciliary 'curl-up' movement, transverse forces (possibly induced by radial spokes) need to be considered.

CONCLUSION: This model provides a platform to investigate various assumptions of dynein activity, facilitating us to evaluate their rationality and propose possible dynein activation patterns.
\end{abstract}

Keywords: Respiratory cilia, dynein, ultrastructure, finite element method

\section{Introduction}

The cilia in the respiratory system consist of a highly conserved structure, whose characteristic architecture is based on a cylindrical arrangement of 9 doublet microtubules surrounding a central pair of singlet microtubules [1] (Fig. 1). The dynein motors located between adjacent doublets convert the chemical energy into mechanical work for doublet sliding. This shear between outer doublets drives the cilia bending and other passive elastic components including the central microtubule couple are involved in the overall regulation, producing the effective ciliary movement.

Due to the inherent difficulties in experimental observations, researchers can hardly investigate the internal mechanics of a moving cilium directly. Alternatively, computational simulations can help to reveal the internal mechanics of the ciliary motion and have therefore attracted considerable interests. Lindemann's geometric clutch theory [2-4] proposes that the transverse force acts as the main regulator of switching and generates the beating motion of the cilia. Satir's switch-point model [5-7] believes that

\footnotetext{
${ }^{*}$ Corresponding author: Duanduan Chen, School of Life Science, Beijing Institute of Technology, Beijing 100086, China. Tel./Fax: +86 106891 2154; E-mail: duanduan@bit.edu.cn.
} 


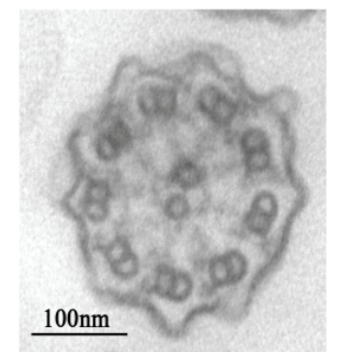

(a)

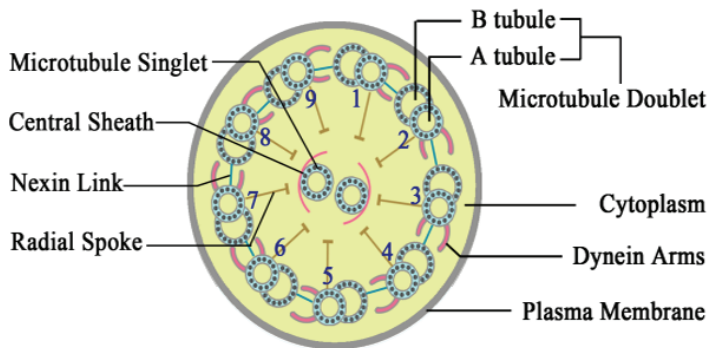

(b)

Fig. 1. The ultrastructure of respiratory cilia. (a) displays the cross-section of a cilium by transmission electron microscopy and (b) is the illustration of the components in ciliary body.

the 9 microtubule doublets work in groups; the switching of active work of the dyneins in the two groups produces the ciliary forward and backward beating. Brokaw [8-10] proposes the curvature-controlled model of ciliary motion. Gueron and colleagues [11] and Teff and others [12] suggest the switching occurs at constant angles or particular positions of the cilia. Moreover, Gueron and Levit-Gurevich [13] propose the first 3D simulation of the ciliary motion based on the ciliary internal $9+2$ structure. More recent work concerning cilia that incorporates discrete representations of the dynein arms, the passive elastic structure of the axoneme, and surrounding fluid dynamics in a 2D system is described by Dillon et al. [14]. Besides, Mitran [15] proposes a 3D model to simulate the respiratory cilia by using largedeflection, curved, finite-element beams. More recently, Chen et al. [16] propose a 3D model to simulate a $9+0$ ciliary ultrastructure - the embryonic cilia. Although it focuses on a different type of cilia, it presents a more realistic model of ciliary ultrastructure and ensures the feasibility to simulate the complex ciliary movement by Finite Element Method. Apart from the computational works, recently, Burgoyne et al. [17] reports the detailed geometric information regarding the ultrastructure of human respiratory cilia, and indicates the difference between human airway cilia to the conventionally studied cilia/flagella, such as Chlamydomonas. This study provides the basis for more realistic modelling of lung ciliary ultrastructure, contributing to future investigations of dynein activity.

The abovementioned studies, among others, improved our understandings regarding the ciliary motility; however, modelling the internal mechanism of cilia is still a very challenging topic. How the ciliary axonemal structure converts the dynein-driven microtubule sliding into smooth and coordinated repetitive waves of bending remains a mystery. In this work, we introduce a 3D model of the ciliary ultrastructure and study the ciliary motion empowered by dynein motors. This model simulates the activity of the dyneins by applying longitudinal forces, in an embedded manner, along the doublet microtubules in an appropriate (temporally and spatially) manner. The hypotheses proposed by early studies regarding the dynein activation pattern is simulated and the possible protein activation pattern for lung cilia is proposed.

\section{Methods}

\subsection{Geometry of the model}

Experimental observations by transmission electron microscopy report that the length of cilia is about 5-7 $\mu \mathrm{m}$ in the trachea [18]. In our model, the length of the cilium is assigned as $6 \mu \mathrm{m}$. As shown in the left picture of Fig. 2(a), the geometrical model of the cilium consists of a cylindrical body and a 


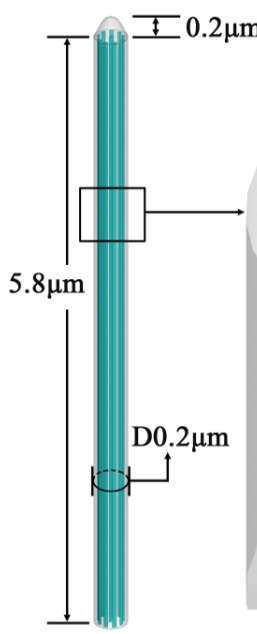

(a)
Microtubule Doublet

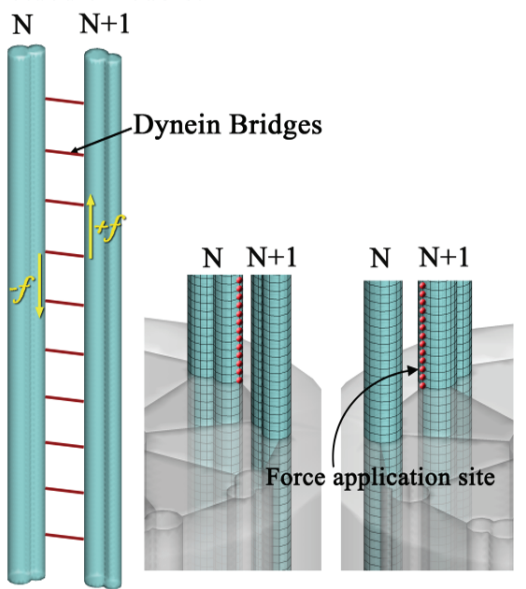

(b)

(c)

Fig. 2. The geometry of the model. (a) displays the geometry of the ciliary model; (b) is the illustration of the mechanical function of dynein motors; and (c) displays the application sites of dynein force in the discretized model.

parabolic tip, the lengths of which are $5.8 \mu \mathrm{m}$ and $0.2 \mu \mathrm{m}$, respectively, and the diameter of the ciliary body is $0.2 \mu \mathrm{m}$ [15]. The ultrastructure of the cilium is modelled according to the reported data in [17] and to Fig. 1(a). The width of a doublet is reported to be 20-30 nm [19]. In this model, the width of the doublet microtubules is assigned as $25 \mathrm{~nm}$. The diameter of the B-tubule as well as the singlet is set to be $16 \mathrm{~nm}$ [17]. The distance between the doublet to the center of the cilium is $60 \mathrm{~nm}$, according to Fig. 1(a) and the transverse sectional image of the cilia in [17].

The spatial resolution of the cilia involves 288 points in the vertical direction (263 points for the cylindrical body and 25 points for the parabolic tip) and 56 points around the circumference. This ensures the interval of the grid points along the longitudinal direction of the doublet microtubules is about $22 \mathrm{~nm}$, which is consistent to the reported repeating distance of inner dynein arms (controlling the beat form) [17]. The cylindrical body of the cilium that contains the microtubules is discretized by linear extrusion from the unstructured bottom mesh of the model; while the parabolic tip is discretized by tetrahedral mesh. The base grid consists of a total of 673,284 cells (a grid independency study is discussed in the sequel).

\subsection{Mechanical properties of the cilium}

The mechanical properties of the axenomal components of the cilium are obtained from literatures, although it should be noted that the data presented in this section is highly affected by the difficulties of the in vivo studies and the precise mechanical properties of ciliary ultrastructure specifically for respiratory cilia are lacking. Results from relevant studies on the Young's modulus of the ciliary mcrotubules vary among 5-9 GPa [20], 1.2 GPa [21], 0.47-0.51 GPa [22], and Kikumoto et al. [23] suggested the rigidity of microtubules is about $7.9 \times 10^{-24} \mathrm{Nm}^{2}$, indicating a mechanical modulus of $0.1 \mathrm{GPa}$ based on the geometry of our model. Those studies are not focused on respiratory cilia specifically, which actually presents a much shorter length and therefore might possess different mechanical properties. In this study, we assume the doublet microtubules present homogeneous and isotropic properties and the elastic modulus is $3 \mathrm{GPa}$; while the elastic modulus for the singlets is $1 \mathrm{GPa}$. The mechanical information of the cytoplasm wrapping the doublets is also lacking. This is partly because of the uncertainty 


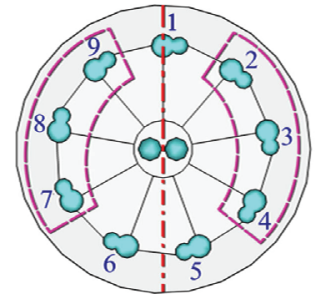

(a)

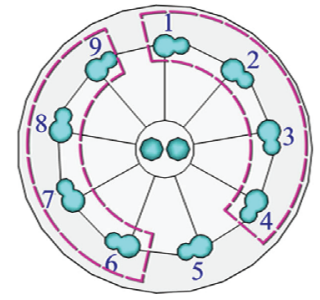

(b)

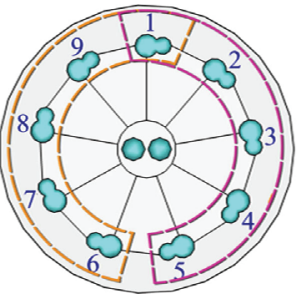

(c)

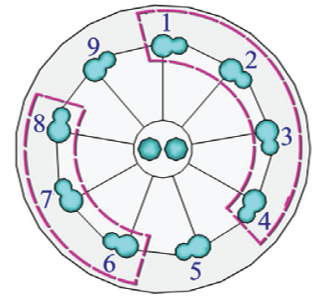

(d)

Fig. 3. The doublet grouping scenarios. (a)-(d) display the doublet grouping plan in scenarios I-IV, respectively. The dashed curves show the doublet groups and the dash-dot line in (a) displays the central line crossing the middle between the singlet pair.

about the exact biochemical components of it and is also due to that the elastic properties of cytoplasm can be largely different in various species, ranging from $25 \mathrm{~Pa}$ to $5 \times 10^{5} \mathrm{~Pa}[24[25]$. In this study, the mediated cytoplasm is modelled as a solid. A parameter study of the elasticity of this solid and their effect on the ciliary movement has been conducted. The elastic modulus of the modelled cytoplasm is finally assigned to be $200 \mathrm{~Pa}$; this value, together with the mechanical properties of microtubules, gives the most flexibility of ciliary movement and is close to the limit of what can be realistically computed in terms of local mesh deformations for the ultrastructure of the cilium. More precise de terminations of the mechanical properties of respiratory ciliary ultrastructure would be pursued in future studies, when detailed experimental estimations are achievable.

\subsection{Theories regulating the dynein activation}

The ciliary beating is powered by the dyneins that anchored at regular intervals along the doublets [26]. With hydrolysis of ATP, the motor protein forms a transient attachment to its adjacent doublet, converting chemical energy into mechanical forces, and thus pushes the target doublet towards the plus end [27|28], as shown in Fig. 2(b). There are hundreds of dynein motors along one doublet microtubule. These protein motors begin to attach to the adjacent doublet in a sequential manner. Since the base cell is the energy source of dynein activation, we assume that the attachment of dynein motors occurs from the bottom of the doublet. When the lower-positioned dynein bridges are formed, they reduce the distance between two adjacent doublets in the near-by region, thus facilitate the upper-positioned dynein bridges to be established. In other words, the dynein attachments between two neighboring doublets occur from the bottom and 'climb up' towards to the ciliary tip.

Previous studies [5 29] propose that, for respiratory cilia, the dyneins located along the 9 doublets should work in two switching groups in order to create the planar beating motion. Based on this assumption, we investigate four scenarios of dynein activity and discuss their rationality. Figure 3 illustrates the scenarios. In scenario I, the two groups of doublets include doublets 2-4 and doublets 7-9, respectively (Fig. 3(a)). This type of division is similar to Lindemann's 'Geometric Clutch' model [2]. In one group of the doublets, the dynein attachments start from bottom towards tip in a linear manner. When this finishes, it triggers the dynein attachments in the other group; at the same time, the dynein bridges in the original group starts to detach from the tip to the bottom. In scenario II, we group the doublets as quaternions, which are doublets 1-4 and doublets 6-9 (Fig. 3(b)); while in scenario III, we group the doublets as pentad, which are doublets 1-5 and doublets 6-1 (Fig. 3(c)). The doublet 1, in this case, is included in both of the groups: when the dyneins in doublets 1-5 are activated, the dynein proteins along doublet 1 pushes doublet 2 towards the ciliary tip; while, when the dyneins in doublets $6-1$ are activated, 
the dynein proteins along doublet 9 pushes doublet 1 towards the tip. Finally in scenario IV, the doublets are grouped according to Satir's model [5], where doublets 1-4 are in one group and doublets 6-8 are with the other group (Fig. 3(d)), presenting an asymmetric grouping pattern. The abovementioned doublet grouping scenarios divide the doublets according to the central line between the singlet pair, as shown in Fig. 3(a). This is due to the reported lung cilia beating pattern, which is nearly within the plane determined by this central line [5].

An activated dynein arm can produce sliding forces between two adjacent doublets. Thus, in our model, we use a pair of point loads exerted on the corresponding positions of the two neighbouring doublets, with same magnitude and in opposite directions, to simulate the mechanical effects induced by a formed dynein bridge. The force exerted by a single arm upon a microtubule has been reported varying from 1-10 $\mathrm{pN}$ [30-32]. In this study, the force generated by a single dynein arm is assigned to be $5 \mathrm{pN}$. The beating frequency of respiratory cilia is reported to be $10.6-17 \mathrm{~Hz}$ [18]. In the current study, we assume the beating frequency of the cilium is $12 \mathrm{~Hz}$. Therefore, it costs $0.0417 \mathrm{~s}$ for the dynein bridges to finish their formation in one group of doublets.

\subsection{Numerical methods}

During computation, the motility of the cilium is calculated by embedding the produced forces on the discretized ciliary elements (properly located in space and time according to the scenarios we discussed in Section 2.3) and solving the resulting problem using a stress solver in CFD-ACE+ (ESI CFD, France), based on the Finite Element Method. This platform allows the concurrent embedding of the forcesgenerating elements of the axoneme, along with the passive deformable elements of the structure into a unified framework, thus permitting the computation of self-induced deformation and motion for this cilium system. This capability is combined together with necessary re-meshing techniques required by the grid deformation. In this study, a Solid-body Elasticity Analogy method [33], where the re-meshing problem is solved by calculating the displacement between the moved boundaries and based on the solution of the equations of linear elasticity, is employed.

\section{Results and discussion}

To evaluate the insensitivity of the simulation results to the resolution, the solution on a finer grid with 2,003,712 cells has been studied (for scenario I) to compare with the results in the base grid. The ciliary motion in both of the resolutions presents the same pattern, and the difference of the maximum ciliary deformation occurring at the ciliary tip is $7.62 \%$. Therefore, for the purposes of our study the base resolution is adequate and is employed in further simulations of other dynein activation scenarios.

\subsection{The bending deformation induced by dynein motors}

As indicated in Section 2.3, the activation of dyneins starts from the bottom of the doublets and climb up towards the ciliary tip. When a dynein bridge is established, it generates sliding movement between the two adjacent doublets by pushing the target (higher-numbered) doublet tipward and dragging the 


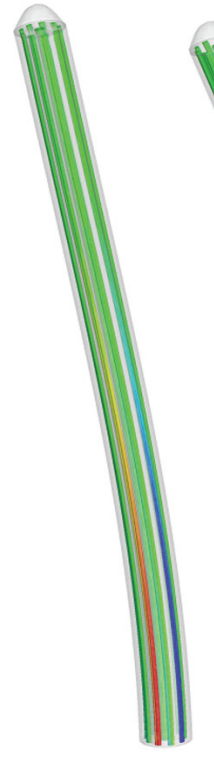

(a)

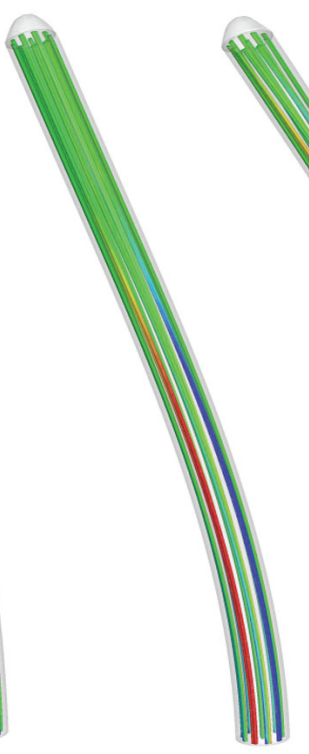

(b)

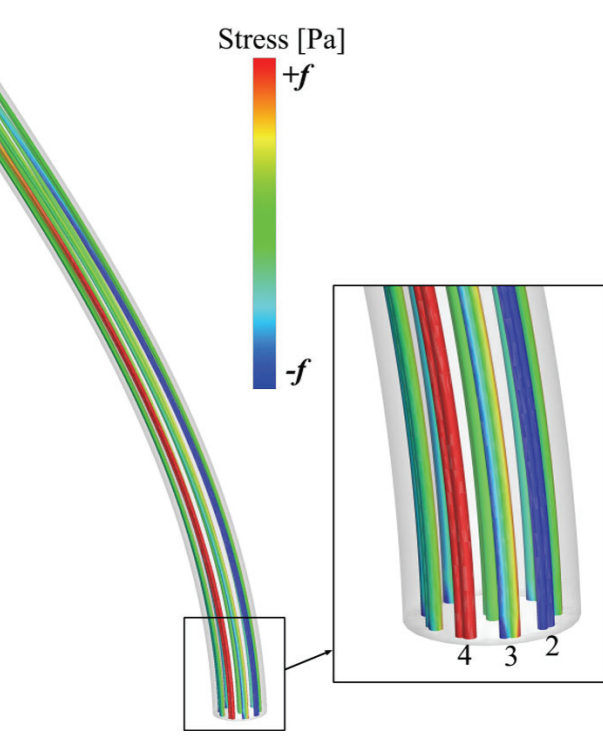

(c)

Fig. 4. The dynein-triggered ciliary bending in scenario I. (a)-(c) show the results when the dynein bridge establishment is progressed to $1 / 3,2 / 3$, and full of the doublet length, respectively. The stress along the longitudinal direction of the doublets is contoured in order to present the mechanical effects by dynein force.

base (lower-numbered) doublet towards the bottom. With the other passive elastic components, such as the cytoplasm, the local sliding produced by the formed dynein bridges induces bending deformation of the entire ciliary body, and with an appropriate arrangement of the activation of the dynein motors, the cilium can produce a beating movement. Figure 4 displays the dynein climbing process in scenario I, when the dynein motors along doublet 2 and 3 are activated. Figures 4(a) and (b) show the results when the dynein bridge establishment is progressed to $1 / 3$ and $2 / 3$ of the doublet length, respectively; while, Fig. 4(c) shows the result when all the dynein motors are activated along the affected doublets. The longitudinal stress is coloured in the picture in order to represent the dynein climbing process. With the dynein bridges being established from bottom to tip, the sliding force between those affected doublets are increasing, and therefore, generates the bending of the entire cilium.

\subsection{The moving pattern of the cilium}

Figure 5 displays the simulated ciliary motion. The program is implemented for four ciliary beating cycles for each scenario, and the results in the last cycle are presented here. The subfigures (a)-(d) present the results for the four scenarios respectively. The original position of the cilium is shown in the images marked by ' 0 '. After the first half cycle, the dynein activity induces the ciliary bending to the maximum at one direction; while, in the next half cycle, with the dynein activity in the other group of doublets begins to work, the cilium, thus, deforms towards the opposite direction. The ciliary motion is finally converged to a certain pattern - the forward and backward beating.

The beating patterns of the cilium in all of the four scenarios are non-symmetric. The maximum deformations occurred at the ciliary tip at the end of effective stroke are $1.17 \mu \mathrm{m}, 1.55 \mu \mathrm{m}, 1.72 \mu \mathrm{m}$, 


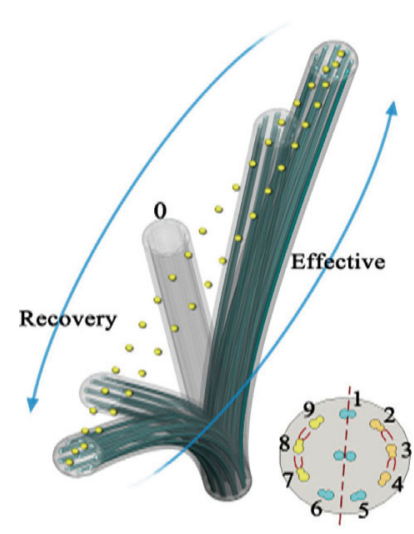

(a)

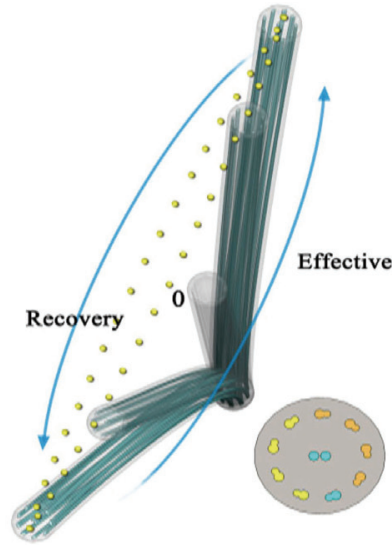

(b)

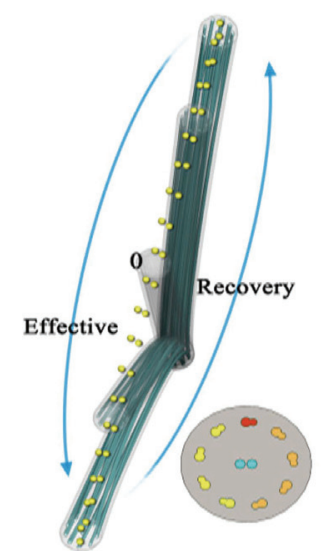

(c)

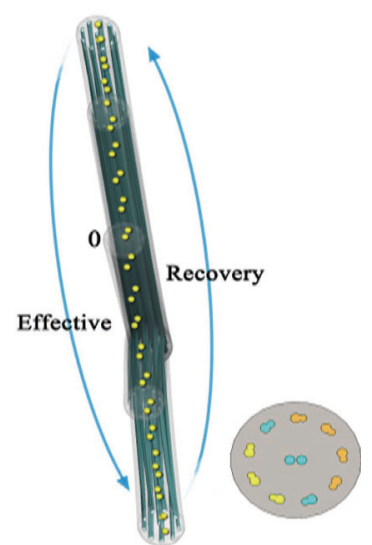

(d)

Fig. 5. The simulation results of this computational model. (a)-(d) show the results of scenarios I-IV, respectively.

and $1.53 \mu \mathrm{m}$, respectively for scenarios I-IV, which are $19.5 \%, 25.8 \%, 28.7 \%, 25.5 \%$ of the ciliary length respectively; and the maximum ciliary deformation at the end of recovery stroke in the four cases are $1.11 \mu \mathrm{m}, 1.48 \mu \mathrm{m}, 1.55 \mu \mathrm{m}$, and $1.15 \mu \mathrm{m}$, respectively. The magnitude of ciliary deformation is dependent on the number of dynein motors involved during the ciliary motion. In scenario III, during each half of the beating cycle, the dynein proteins along four doublets are activated together, which, therefore, is able to generate larger ciliary deformations than other cases. The difference of the maximum deformation during effective and recovery strokes for scenarios I-IV are 5.4\%, 4.73\%, $11.0 \%$, and $33.0 \%$. During the effective stroke in scenario IV, the dyneins along three doublets (doublet 1, 2 and 3) are activated; while, during the recovery stroke, only the dyneins along doublet 6 and 7 are activated, which induces the higher difference of the maximum deformation between the two phases in scenario IV. Moreover, the direction of the effective stroke (determined by the maximum deformation of the cilium) in scenarios I and II is opposite to that in scenarios III and IV. In cases I and II, the effective stroke is towards the side of doublet 1 ; while, in cases III and IV, the effective stroke is towards the side of doublet 5 or 6 . Unfortunately, to the best of our knowledge, there is no observation reports clarified the specific direction of effective stroke for airway cilia; therefore, the resulted direction of effective stroke cannot be used to evaluate the rationality of dynein activation hypotheses.

Considering the reference plane that passes doublet 1 and is perpendicular to the plane determined by the two singlets, the ciliary movements presented in the four scenarios are nearly along this reference plane but show various small derivation angles. The maximum deviation angle of the cilium during recovery stroke comparing to the position of the cilium at the beginning of power stroke is $13.4^{\circ}, 12.1^{\circ}$, $3.5^{\circ}$ and $2.6^{\circ}$, respectively for scenarios I-IV. Previous experimental study [34] shows that this angle should be less than $5^{\circ}$, indicating the dynein activation patterns in scenarios III and IV can present more reasonable results. Observation evidence to reveal the actual moving pattern of respiratory cilia is lacking. Previous illustrations of lung cilia beating indicated that the maximum deformation of the cilium, measured at the ciliary tip, during the effective and recovery strokes may present a difference between 10\%-25\%, derived from [35]. Both of the results in scenarios III and IV are close to this difference range (11.0\% and $33.0 \%$ respectively for cases III and IV), indicating the rationality of these models; while, in scenario III, the dyneins of all of the outer doublets are involved and the simulated deformation difference between effective and recovery strokes is just within the range derived by [35]. 
It is generally believed that the dynein forces are the main reason for ciliary motility and may determine the deformation magnitude of the cilia [36]. As shown in our results, the grouping assumption can indeed produce the planar beating of cilia. However, since the motivation force is just along the longitudinal direction of the microtubules, the generated beating motion is made of ciliary bending; the "curl up' motion presented during the recovery stroke as in observations cannot be produced. This indicates that, apart from the longitudinal force, to present the realistic ciliary motion, the transverse force (may be generated by the radial spokes) should be considered, which is also the next step of this computational work.

\section{Conclusions}

The hypothesis that the dynein proteins along doublet microtubules may work in groups has been proposed a long time ago. However, it is very difficult to be confirmed by experiments; researchers are lack of knowledge about dynein activity and cannot fully manipulate them. Three-dimensional modelling of the ciliary ultrastructure and simulating the dynein activity is an alternative way to confirm this theory. Therefore, we establish this mathematical model, including the $9+2$ microtubule structure, the cytoplasm and the dynein motors, to test various dynein-grouping assumptions. Our results reveal that the switching activation of dyneins is able to produce the planar beating movement of the cilium, and the forward and backward motion of the cilium is asymmetric. Based on the limited geometric information of the beating motion produced by airway cilia, the results in scenario III present the most reasonable ciliary beating pattern, which involves the active dynein works along most of the doublets and presents $11 \%$ of the difference of the deformation magnitude between the effective and recovery stroke and $3.5^{\circ}$ of the derivation angle.

Ciliary motility in human respiratory system has drawn much attention in recent years [37]. It is closely related to the health of respiratory system and may indirectly influence the function of other organs [38]. Revealing the protein-controlled mechanism of ciliary motion is crucial to understand the system. The current study based on reported geometric and functional data of ciliary ultrastructure and motor proteins is not the most accurate, but it confirms the feasibility of the algorithms to be applied in cilia studies and simulates ciliary motion induced by dynein motors alone, which may contribute to our understandings of this complex protein-microtubule system.

\section{Acknowledgements}

This study is supported by the National Natural Science Foundation of China (no. 31200704 and 81471752). We would like to acknowledge the ESI Group and Mr. Xiangde Zhu for allowing us to use CFD-ACE+, and thank Ms. Yujiao Zhou for providing the transmission electron microscopic image in Fig. 1(a).

\section{References}

[1] Satir, P. and M.A. Sleigh, The physiology of cilia and mucociliary interactions. Annu Rev Physiol 1990; 52: 137-55.

[2] Lindemann, C.B., Geometric Clutch model version 3: the role of the inner and outer arm dyneins in the ciliary beat. Cell 
Motil Cytoskeleton 2002; 52 (4): 242-54.

[3] Lindemann, C.B., Testing the geometric clutch hypothesis. Biol Cell 2004; 96 (9): 681-90.

[4] Lindemann, C.B., The geometric clutch as a working hypothesis for future research on cilia and flagella. Ann N Y Acad Sci 2007; 1101: 477-93.

[5] Satir, P. and T. Matsuoka, Splitting the ciliary axoneme: implications for a "switch-point" model of dynein arm activity in ciliary motion. Cell Motil Cytoskeleton 1989; 14 (3): 345-58.

[6] Satir, P., The role of axonemal components in ciliary motility. Comp Biochem Physiol A Comp Physiol 1989; 94 (2): 351-7.

[7] Satir, P., T. Hamasaki, and M. Holwill, Modeling outer dynein arm activity and its relation to the ciliary beat cycle. Cilia, Mucus, and Mucociliary Interactions. New York: Marcel Dekker; 1998.

[8] Brokaw, C.J., Thinking about flagellar oscillation. Cell Motil Cytoskeleton 2009; 66 (8): 425-36.

[9] Brokaw, C.J., Computer simulation of flagellar movement VIII: coordination of dynein by local curvature control can generate helical bending waves. Cell Motil Cytoskeleton 2002; 53 (2): 103-24.

[10] Brokaw, C.J., Computer simulation of flagellar movement IX. Oscillation and symmetry breaking in a model for short flagella and nodal cilia. Cell Motil Cytoskeleton 2005; 60 (1): 35-47.

[11] Gueron, S., et al., Cilia internal mechanism and metachronal coordination as the result of hydrodynamical coupling. Proc Natl Acad Sci U S A 1997; 94 (12): 6001-6.

[12] Teff, Z., Z. Priel, and L.A. Gheber, The forces applied by cilia depend linearly on their frequency due to constant geometry of the effective stroke. Biophys J 2008; 94 (1): 298-305.

[13] Gueron, S. and K. Levit-Gurevich, A three-dimensional model for ciliary motion based on the internal $9+2$ structure. Proc Biol Sci 2001; 268 (1467): 599-607.

[14] Dillon, R.H. and L.J. Fauci, An integrative model of internal axoneme mechanics and external fluid dynamics in ciliary beating. J Theor Biol 2000; 207 (3): 415-30.

[15] Mitran, S.M., Metachronal wave formation in a model of pulmonary cilia. Comput Struct 2007; 85 (11-14): 763-774.

[16] Chen, D., D. Norris, and Y. Ventikos, Ciliary behaviour and mechano-transduction in the embryonic node: computational testing of hypotheses. Med Eng Phys 2011; 33 (7): 857-67.

[17] Burgoyne, T., et al., Generation of a three-dimensional ultrastructural model of human respiratory cilia. Am J Respir Cell Mol Biol 2012; 47 (6): 800-6.

[18] Ho, J.C., et al., The effect of aging on nasal mucociliary clearance, beat frequency, and ultrastructure of respiratory cilia. Am J Respir Crit Care Med 2001; 163 (4): 983-8.

[19] Downing, K.H. and H. Sui, Structural insights into microtubule doublet interactions in axonemes. Curr Opin Struct Biol 2007; 17 (2): 253-9.

[20] Baba, S.A., Flexural rigidity and elastic constant of cilia. J Exp Biol 1972; 56 (2): 459-67.

[21] Bronzino, J., The biomedical engineering handbook - Biomedical Engineering fundamentals. Florida: CRC Press; 2006.

[22] Venier, P., et al., Analysis of microtubule rigidity using hydrodynamic flow and thermal fluctuations. J Biol Chem 1994; 269 (18): 13353-60.

[23] Kikumoto, M., et al., Flexural rigidity of individual microtubules measured by a buckling force with optical traps. Biophys J 2006; 90 (5): 1687-96.

[24] Parsegian, V.A., N. Fuller, and R.P. Rand, Measured work of deformation and repulsion of lecithin bilayers. Proc Natl Acad Sci U S A 1979; 76 (6): 2750-4.

[25] Byfield, F.J., et al., Cholesterol depletion increases membrane stiffness of aortic endothelial cells. Biophys J 2004; 87 (5): 3336-43.

[26] Gibbons, I.R. and A.J. Rowe, Dynein: A Protein with Adenosine Triphosphatase Activity from Cilia. Science 1965; 149 (3682): 424-6.

[27] Fox, L.A. and W.S. Sale, Direction of force generated by the inner row of dynein arms on flagellar microtubules. J Cell Biol 1987; 105 (4): 1781-7.

[28] Summers, K.E. and I.R. Gibbons, Adenosine triphosphate-induced sliding of tubules in trypsin-treated flagella of seaurchin sperm. Proc Natl Acad Sci U S A 1971; 68 (12): 3092-6.

[29] Lindemann, C.B., Experimental evidence for the geometric clutch hypothesis. Curr Top Dev Biol 2011; 95: 1-31.

[30] Minoura, I., T. Yagi, and R. Kamiya, Direct measurement of inter-doublet elasticity in flagellar axonemes. Cell Struct Funct 1999; 24 (1): 27-33.

[31] Shingyoji, C., et al., Dynein arms are oscillating force generators. Nature 1998; 393 (6686): 711-4.

[32] Schmitz, K.A., et al., Measurement of the force produced by an intact bull sperm flagellum in isometric arrest and estimation of the dynein stall force. Biophys J 2000; 79 (1): 468-78.

[33] Johnson, A.A. and T.E. Tezduyar, Mesh update strategies in parallel finite-element computations of flow problems with moving boundaries and interfaces. Comput Methods Appl Mech Eng 1994; 119 (1-2): 73-94.

[34] Chilvers, M.A. and C. O'Callaghan, Analysis of ciliary beat pattern and beat frequency using digital high speed imaging: comparison with the photomultiplier and photodiode methods. Thorax 2000; 55 (4): 314-7. 
[35] Sanderson, M.J. and M.A. Sleigh, Ciliary activity of cultured rabbit tracheal epithelium: beat pattern and metachrony. J Cell Sci 1981; 47: 331-47.

[36] King, S.M., A solid-state control system for dynein-based ciliary/flagellar motility. J Cell Biol 2013; 201 (2): 173-5.

[37] Chang, J.H., et al., Dietary polyphenols affect MUC5AC expression and ciliary movement in respiratory cells and nasal mucosa. Am J Rhinol Allergy 2010; 24 (2): e59-62.

[38] Romanelli, M.C., et al., Nasal ciliary motility: a new tool in estimating the time of death. Int J Legal Med 2012; 126 (3): 427-33. 